\title{
EXPERIMENTAL AND NUMERICAL RESULTS OF SLOSHING WITH CRYOGENIC FLUIDS
}

\section{J. Lacapere, B. Vieille, and B. Legrand}

The paper presents different sloshing experiments and computations performed with cryogenic liquids. Heat and mass transfer at the interface during sloshing phenomenon is responsible of large pressure variation in the vessel. This work helps understanding the behavior of thermal destratification and the associated pressure decay. Comprehension of this phenomenon is crucial for future space missions with ballistic phases where free surface will not be flat. These sloshing experiments can be used for validating numerical models taking into account heat and mass transfer at the interface.

\section{INTRODUCTION}

Prediction of temperature and pressure evolution in cryogenic tanks of space launchers is mandatory for actual missions if one wants to optimize propellant and pressurant gas masses. During propulsive phases, sloshing could occur in tanks with different pressure responses in hydrogen and oxygen upper stage tanks. Future launchers with ballistic phases will be also submitted to flight phases with strong perturbations of the liquid/gas interface. Like during sloshing, these dynamic perturbations and the resulting free surface movements will be linked to thermal destratification of liquid and gas phases and thus to strong pressure variations. In order to understand these destratification phenomena, some sloshing experiments with cryogenic fluids have been performed through the French-German COMPERE (COMPortement des ERgols dans les REservoirs) program.

Destratification phenomenon and the impact on pressure evolution have been studied experimentally using mixing-induced fluid destratification experiments [1]. As for destratification due to sloshing and related pressure evolution, these issues have not been studied extensively even despite their importance for space launchers.

From a dynamic point of view, the sloshing phenomenon is now quite well understood on earth or at low gravity. It has been studied extensively since 1960s $[2,3]$. Some antisloshing devices are thus well defined in tanks in order 
to limit pressure loads on tank walls and perturbations on the spacecraft stability [4-6]. However, sloshing experiments with cryogenic fluids (with monitoring the associated pressure evolution) have not been performed extensively by space launcher community. Moran [7] performed some sloshing experiments with liquid hydrogen for National Aero-Space Plane (NASP) project and pointed out the importance of this phenomenon for future space missions with "greater bulk fluid motion" and the necessity to have "computational fluid dynamic techniques... to accurately predict pressure and temperature conditions."

Presented in this paper are different sloshing experiments performed with liquid nitrogen and liquid oxygen with the objective of better understanding the thermohydraulic behavior and obtaining data for numerical validations. Some numerical computations associated with the sloshing tests are also presented. These numerical tools could be useful for predicting complex thermohydraulic phenomena occurring during future ballistic phases and corresponding transient phases (engine shut-down, separation phases, reorientation phases, etc.).

\section{TESTS DESCRIPTION}

\subsection{Description of the Test Bench}

Experimental tests have been performed in 2002 with liquid nitrogen and in 2005 with liquid oxygen using the same cryostat. They have been performed in the Advanced Technical Division of Air Liquide. The cryostat used for the sloshing experiments was made of glass for observing free surface evolution (Fig. 1). For limiting external heat fluxes, it was made with a double vacuum-insulated wall. Internal dimensions were a radius of $19 \mathrm{~cm}$ and a height of $80 \mathrm{~cm}$.

This cryostat was instrumented with a capacitance probe in order to get the level of the free surface and with about 10 temperature sensors to measure thermal stratification in the liquid and in the vapor. Two pressure sensors were also added at the bottom and at the top of the cryostat for measuring pressure evolution during sloshing.

The same cryostat with internal equipment was also used for depressurization experiments performed in 2002 in the frame of COMPERE program.

For sloshing experiments, the cryostat was installed on a moving table for applying sinus lateral acceleration with a specific amplitude and frequency.

The cryostat was also connected to external pressurization systems: either vapor pressurization (nitrogen and oxygen for the tests performed, respectively, in 2002 with liquid nitrogen and in 2005 with liquid oxygen), either helium pressurization (helium is considered as a noncondensable gas widely used in cryogenic propulsion). 


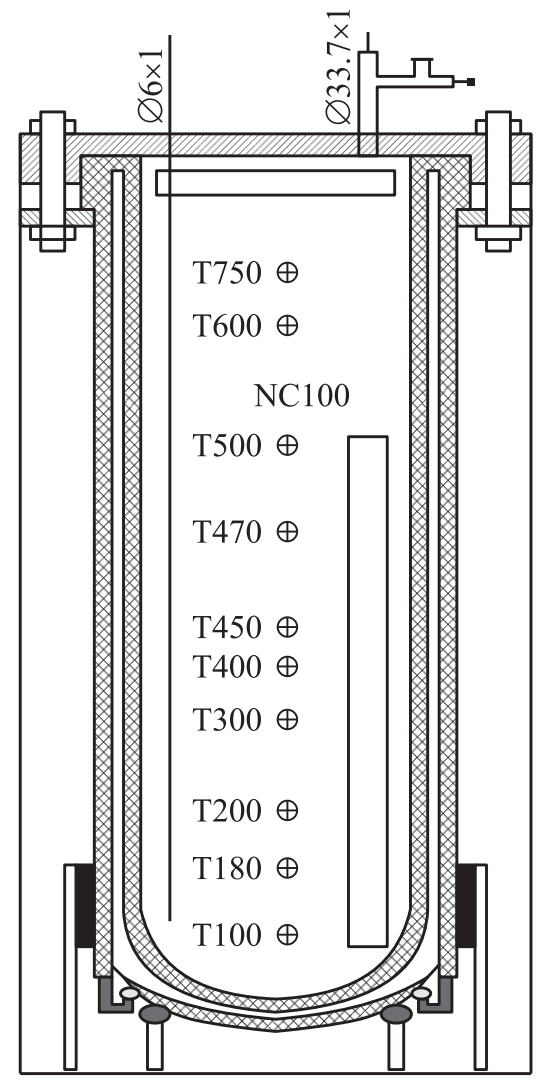

Figure 1 Schematic of the cryostat with the double vacuum-insulated wall and internal temperature sensors (dark circles) and the position of the level sensor (vertical rectangle). Internal diameter: $19 \mathrm{~cm}$; internal lenth: $800 \mathrm{~cm}$. Dimensions are in centimeters

Before starting sloshing experiments at the moving table, the cryostat was filled with cryogenic liquid and pressurized either by external gas (vapor or noncondensable gas), or self-pressurized. As the cryostat is made of glass, thermal heat fluxes are important enough to create free convection and natural stratification in the liquid and gas phases.

\subsection{Test Procedure}

The cryostat, open at atmospheric pressure, is filled at a desired level (about $55 \%$ of the total volume). When the pressure in the cryostat is stabilized, it is 
closed and pressurized up to the desired value (about 2.5 bar). When the right pressure value is reached, shaking is initiated at the preset frequency and the amplitude is increased to a desired value (within the first second).

Shaking is maintained during the time needed to get a stable pressure. This stabilization is achieved within the first $100 \mathrm{~s}$.

Thus, two different stratification patterns are created due to two different pressurization techniques applied before sloshing:

(1) vapor pressurization (either nitrogen $-\mathrm{GN}_{2}$, or oxygen $-\mathrm{GOx}$ ), which creates a very strong thermal gradient within a very small liquid layer at the interface. Pressurization up to 2.5 bar was performed within $100 \mathrm{~s}$; and

(2) helium pressurization (GHe), which creates a small thermal gradient within a very small liquid layer. Pressurization up to 2.5 bar was performed in this case within $50 \mathrm{~s}$.

The sloshing tests with liquid oxygen have been performed 3 years later the tests with liquid nitrogen. Therefore, the tools inside (temperature sensors, capacitance probes, etc.) and outside the tank (pressurization line, helium capacity, sonic orifice, etc.) were not exactly the same in the two experiments.

\subsection{Test Parameters}

The following test parameters were varied:

- Two different cryogenic fluids have been tested: liquid nitrogen $\left(\mathrm{LN}_{2}\right)$ and liquid oxygen $(\mathrm{LOx})$;

- Two different pressurization techniques were used resulting in two different thermal stratification patterns and two different ullage compositions;

- Different shaking parameters were used leading to strong or smooth free surface deformations.

This paper is focused only on sloshing close to the first frequency of the cryostat and with vapor pressurization. In fact, it is focused only on the destratification phenomenon of the previously stratified liquid layer and the associated pressure decrease. Sloshing is performed close to the first frequency of the cryostat which is around $2.1 \mathrm{~Hz}$.

Shaking is a sinus excitation with 3-millimeter amplitude and 2.1-hertz frequency.

Two sloshing experiments are thus presented in this paper with only one excitation mode (frequency $2.1 \mathrm{~Hz}$ and amplitude $3 \mathrm{~mm}$ ): One with $\mathrm{LN}_{2}$ pressurized up to 2.5 bar with $\mathrm{GN}_{2}$ and the other with LOx pressurized up to 2.5 bar with GOx. 


\section{NUMERICAL APPROACH}

\subsection{Description of Numerical Models}

The FLUENT computational fluid dynamics (CFD) code is used for these computations with the Volume of Fluid (VOF) model to simulate sloshing. The "geo-reconstructor" scheme is used in order to accurately represent the free surface behavior.

Surface tension effects are not present in these computations, but these effects could be taken into account if needed (e.g., when Bond number is less than 10).

Using the VOF model, FLUENT is capable of solving the governing equations with average properties values at the interface. Therefore, a modification was made to the FLUENT through User Defined Functions (UDF) for taking into account mass transfer between liquid and gas phases. In the sloshing cases presented herein, the condensation phenomenon at the interface occurring during destratification is the major phenomenon explaining ullage pressure evolution and such an extension to FLUENT is mandatory to simulate cryogenic sloshing.

Liquid phase is supposed to be thermally-expanding: liquid density is taken as a function of temperature. Gas phase is supposed to follow the ideal gas law: density is taken as a function of temperature and pressure.

Second-order discretization is used for momentum and energy equations.

The numerical models are three-dimensional (3D), but with the symmetry plane which is coherent with the sloshing axis.

To impose sloshing, source terms (forces) in the momentum equations, obtained from displacement imposed by the moving table, are added using the UDF.

\subsection{Mesh and Iinitial Conditions}

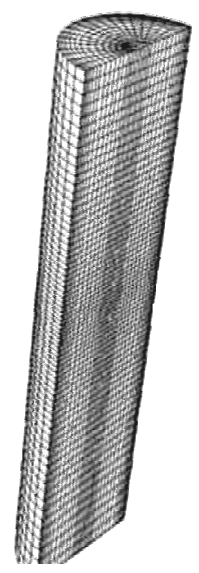

Figure 2 Computational mesh of a half of the cryostat $(\sim 20000$ hexahedral cells)

The cryostat is modeled without internal equipment and without solid walls.

A very simple model is used consisting of a half-a-cylinder (only half of the geometry is modeled). The mesh contains nonuniform hexahedral cells with refined cells close to the interface and coarser cells at the bottom and at the top of the computational domain (Fig. 2). Cells at the initial interface have a size about $3 \mathrm{~mm}$ whereas the cells at the bottom and top have a size about $10 \mathrm{~mm}$. 


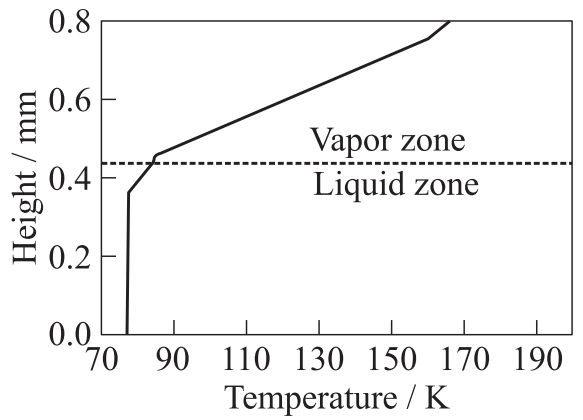

(a)

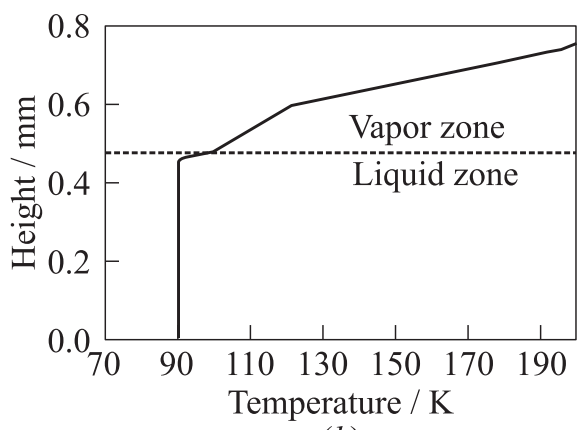

(b)

Figure 3 Initial stratification in the cryostat just before sloshing for $\mathrm{LN}_{2}$ pressurized with $\mathrm{GN}_{2}(a)$ and LOx pressurized with GOx $(b)$

Even if free convection is present in the cryostat, it is not modeled in those computations as the pressure impact is considered negligible during $100 \mathrm{~s}$ of sloshing. Thus, the walls are considered adiabatic.

The computations begin with the beginning of shaking perturbations. Preceding pressurization is not modeled but the impact of this pressurization is taken into account through the initial thermal stratification (from test data).

Initial thermal stratification and initial pressure in the tank before sloshing are imposed in the computations by interpolating the values obtained from experimental tests after pressurization (just before sloshing).

As an example, the initial thermal stratification is shown in Fig. 3 for liquid nitrogen (Fig. $3 a$ ) pressurized with nitrogen vapor $\left(\mathrm{LN}_{2} / \mathrm{GN}_{2}\right)$ up to 2.5 bar and for liquid oxygen (Fig. 3b) pressurized with oxygen vapor ( $\mathrm{LOx} / \mathrm{GOx})$ up to 2.5 bar.

The thermal stratifications exhibit a very strong gradient near the free surface: large temperature variation in a very thin layer. Note that the thermal stratification

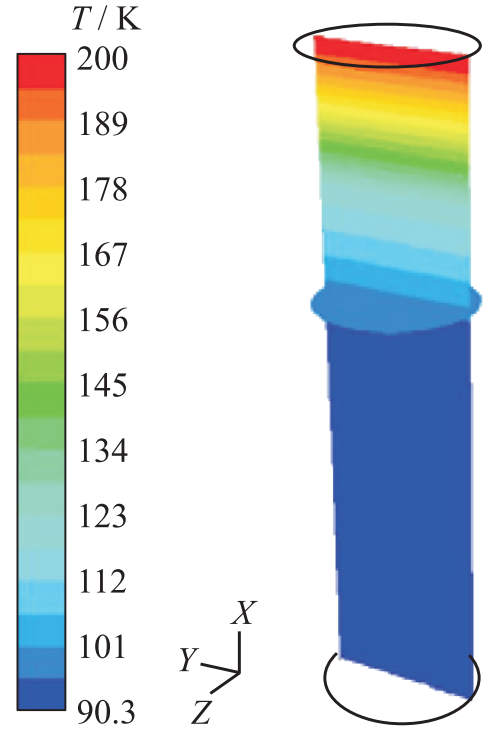

Figure 4 Visual representation (on the symmetry plane and on the free surface) of the initial thermal stratification in the cryostat ( $\mathrm{LOx} / \mathrm{GOx}$ ) before sloshing. (Refer Lacapere et al., p. 272.) 


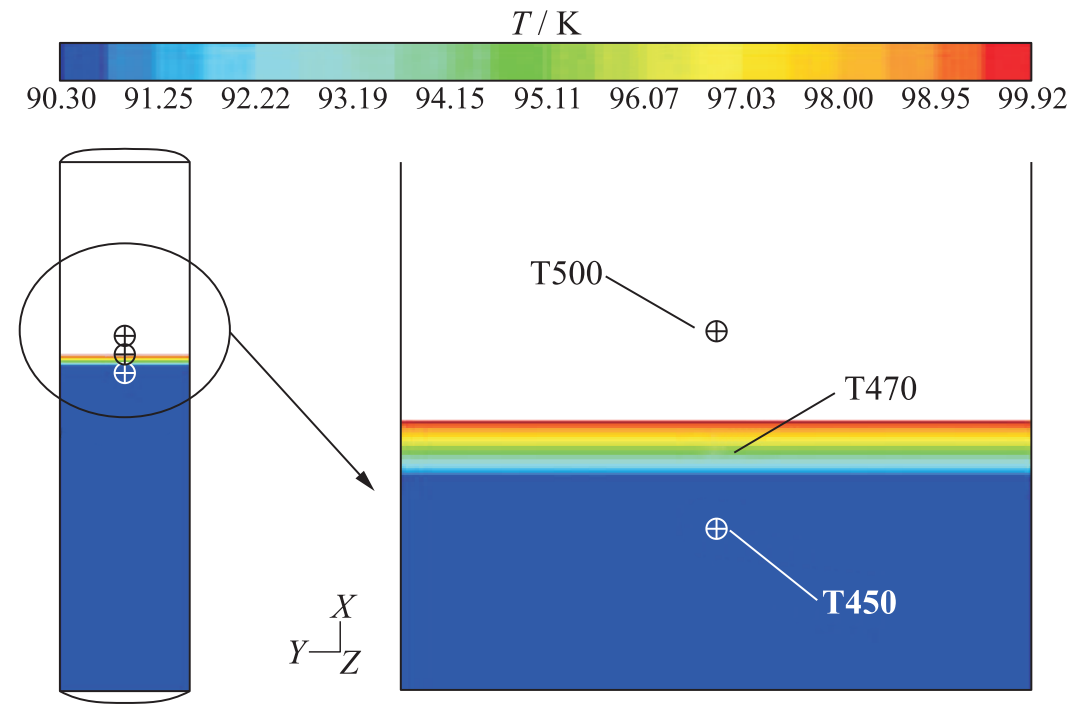

Figure 5 Visual representation of the initial thermal stratification in the liquid phase only in the cryostat ( $\mathrm{LOx} / \mathrm{GOx}$ ). Points correspond to positions of temperature sensors. (Refer Lacapere et al., p. 273.)

of LOx is very strong because of the very rapid pressurization phase (as compared to the $\mathrm{LN}_{2}$ case).

Figure 4 shows an example of the corresponding initial stratification imposed in numerical computations before the beginning of sloshing. In Fig. 5, different temperature scaling is used to visualize the thermal gradient in the liquid phase near the free surface $(\mathrm{LOx} / \mathrm{GOx})$.

\section{RESULTS AND DISCUSSION}

Sloshing occurs in the stable region close to the first cryostat frequency leading to large-amplitude waves rather than to breaking waves with droplets ejections (Fig. 6).

\subsection{Thermal Destratification}

Destratification phenomenon is clearly monitored during sloshing tests due to different temperature sensors in the liquid phase and more precisely near the free surface (see Fig. 7 for temperature evolutions during LOx/GOx sloshing). 


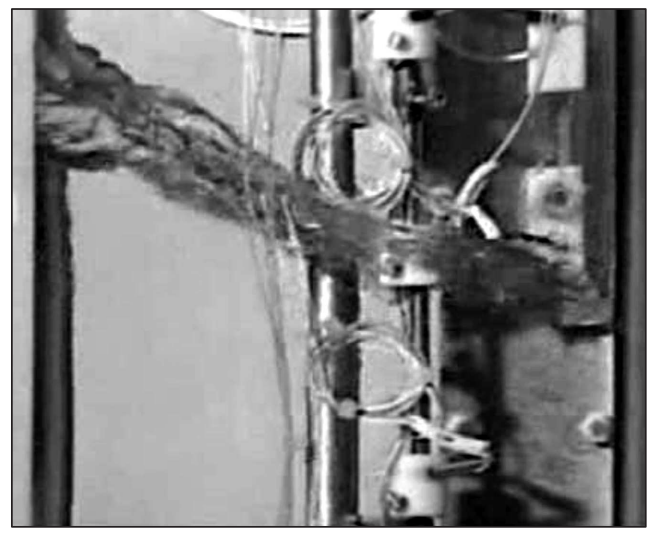

Figure 6 Visualization of experimental free surface deformation during sloshing experiment $\left(\mathrm{LN}_{2}\right)$

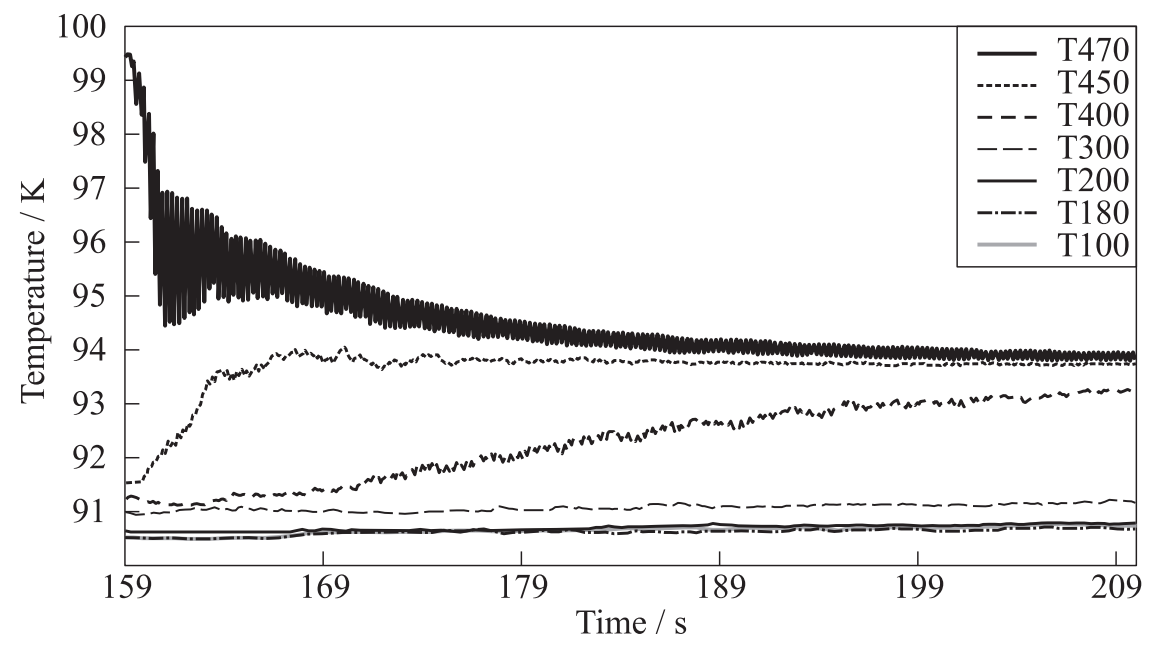

Figure 7 Evolution of temperature in the liquid phase during sloshing experiment (LOx/GOx)

Shaking of the cryostat begins at time $159 \mathrm{~s}$.

The temperature sensors are located at 100, 180, 200, 300,400,450, and $470 \mathrm{~mm}$ from the bottom of the cryostat. Initial liquid level is set at $472 \mathrm{~mm}$ (just above the temperature sensor T470).

The initial thermal stratification is destroyed within first $50 \mathrm{~s}$ of sloshing. The temperature gradient becomes very smooth and the liquid layer becomes thick. 


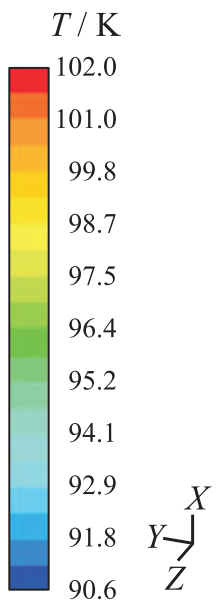

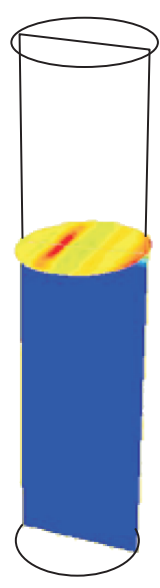

$$
t=0.2 \mathrm{~s}
$$

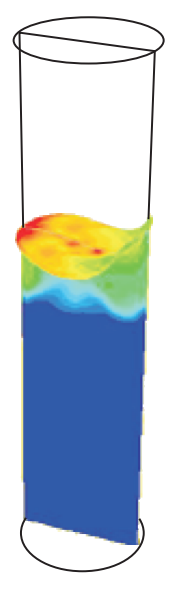

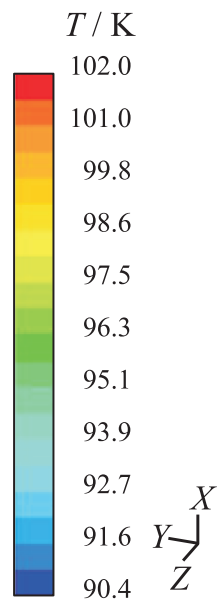

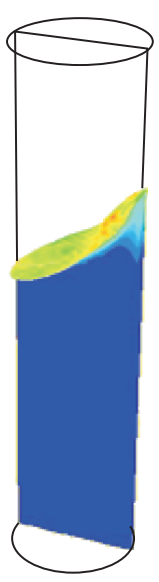

$$
t=4.2 \mathrm{~s}
$$

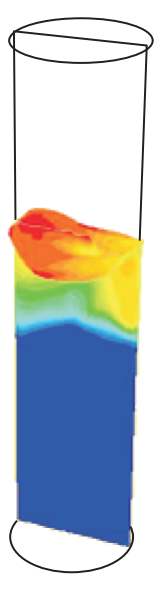

$$
t=44.2 \mathrm{~s}
$$

Figure 8 Time evolution of thermal stratification in the liquid phase - visualization in the symmetry plane and on the free surface, CFD results (LOx/GOx). (Refer Lacapere et al., p. 275.) 


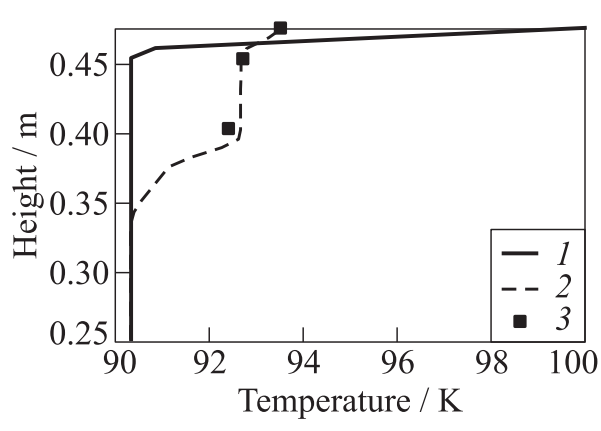

Figure 9 Thermal stratification in the liquid phase (before (1) and after $50 \mathrm{~s}$ of sloshing LOx/GOx: 2 - numerical and 3 - experimental results) ous. The free surface temperature has decreased. The experimental and numerical results on thermal destratifications are in good agreement. The vapor phase condensation is thus well computed by the numerical model.

\subsection{Pressure Evolution}

Figure 10 shows pressure histories obtained in experimental and numerical tests. Two different sloshing experiments are compared for two different fluids $\left(\mathrm{LN}_{2}\right.$ pressurized with $\mathrm{GN}_{2}$ and LOx pressurized with $\mathrm{GOx}$ ) with the same lateral excitation: frequency $2.1 \mathrm{~Hz}$ and amplitude $3 \mathrm{~mm}$.

The pressure histories in the experiments and in the numerical computations are in very good agreement for both liquid nitrogen and liquid oxygen experiments.

It is seen from Fig. 10 that the pressure drop is the fastest during the first $15 \mathrm{~s}$ of sloshing (from 2.5 down to $1.7 \mathrm{bar}$ ). Pressure stabilizes then at about 1.5 bar.

The pressure decay is more important in the very first seconds of sloshing for the LOx tank initially pressurized with GOx because of the initial stratification in the liquid. This initial stratification in LOx was strong (compared to initial stratification in $\mathrm{LN}_{2}$ ) (see Figs. 3 and 5). This stronger initial stratification in LOx has been created due to a faster pressurization phase.

Note that the observed pressure histories have nothing to do with the pressure decay occurring during thrust phases of launcher upper stages (initial stratifications are not so strong and wave amplitude is not so important). 


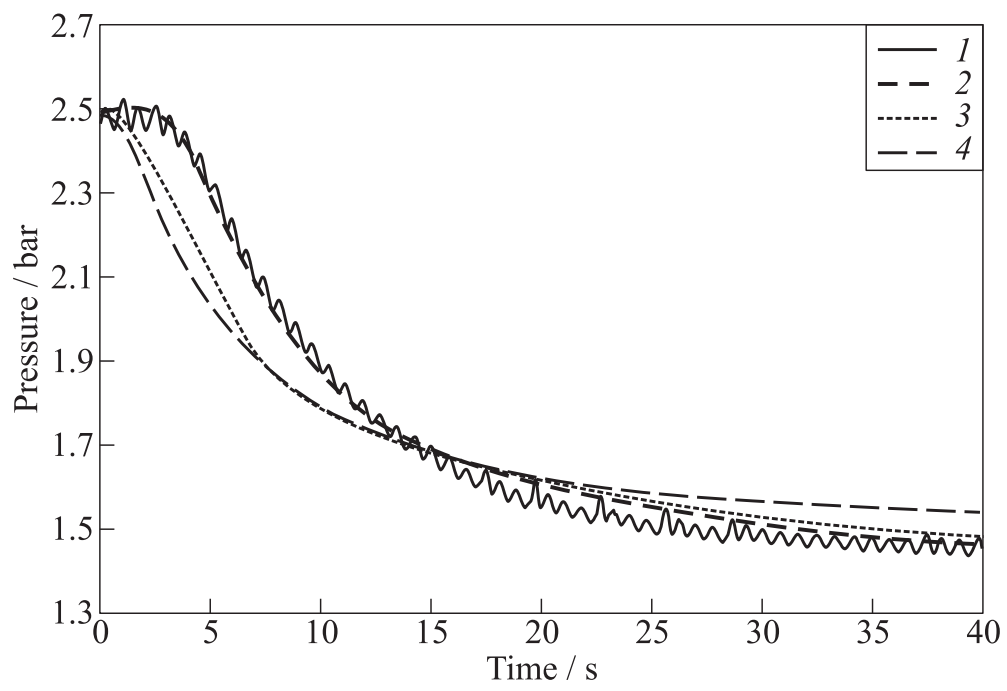

Figure 10 Comparison of measured and calculated pressure histories in the cryostat for $\mathrm{LN}_{2} / \mathrm{GN}_{2}$ and $\mathrm{LOx} / \mathrm{GOx}$ sloshing: $1-\mathrm{LN}_{2}$ sloshing after $\mathrm{GN}_{2}$ pressurization experimental results; $2-\mathrm{LN}_{2}$ sloshing after $\mathrm{GN}_{2}$ pressurization - CFD results; 3 LOx sloshing after GOx pressurization - CFD results; and 4- LOx sloshing after GOx pressurization - experimental results

\section{CONCLUDING REMARKS}

The destratification process in the liquid phase is represented in terms of the comparison between experimental sloshing tests with cryogenic fluids and numerical results. The destratification process is shown to be responsible for a large pressure drop in the cryostat.

The pressure and temperature histories in the fluids are well predicted by the numerical model. The numerical model could also be used in the future for computing pressure and temperature evolution in future upper-stage cryogenic tanks submitted to different external perturbations (engine shut-down, separation phase, reoriention before reignition, etc.).

\section{REFERENCES}

1. Meserole, J.S., O.S. Jones, and A.S. Fortini. 1987. Mixing-induced fluid stratification and ullage condensation. NASA. Lewis Research Center Microgravity Fluid Management Symposium Proceedings. 101-117. 
2. Abramson, H.N. 1966. The dynamic behaviour of liquids in moving containers. NASA SP 106.

3. Dodge, F. T. 1991. Analysis of small amplitude low gravity sloshing in axisymmetric tanks. Microgravity Sci. Technol. 4:228-34.

4. Stephen, D. G. 1972. Liquid-propellant dynamics and suppression. NASA Space Shuttle Technology Conference Proceedings 139-74.

5. Kannapel, M. D. 1987. Liquid oxygen sloshing in space shuttle external tank. AIAA Paper No. 87-2019.

6. Lung, R. J. 1990. Slosh wave excitation and stability of spacecraft fluid systems. TABES Paper 90-1810.

7. Moran, M.E., N.B. Mc Nelis, M. T. Kudlac, and M.S. Haderbusch, and G. A. Satornino. 1994. Experimental results of hydrogen slosh in a 62 cubic foot (1750 liter) tank. AIAA Paper No. 94-3259. 